\title{
Quantum non-Gaussianity witnesses in phase space
}

\author{
Catherine Hughes, ${ }^{1}$ Marco G. Genoni, ${ }^{2,1}$ Tommaso Tufarelli, ${ }^{1}$ Matteo G. A. Paris, ${ }^{3}$ and M. S. Kim ${ }^{1,4}$ \\ ${ }^{1}$ QOLS, Blackett Laboratory, Imperial College London, London SW7 2BW, United Kingdom \\ ${ }^{2}$ Department of Physics \& Astronomy, University College London, Gower Street, London WC1E 6BT, United Kingdom \\ ${ }^{3}$ Dipartimento di Fisica, Università degli Studi di Milano, I-20133 Milano, Italy \\ ${ }^{4}$ School of Computational Sciences, Korea Institute for Advanced Study, Hoegiro 87, Dongdaemun, Seoul 130-722, Korea
}

(Received 28 March 2014; published 10 July 2014)

\begin{abstract}
We address detection of quantum non-Gaussian states, i.e., nonclassical states that cannot be expressed as a convex mixture of Gaussian states, and present a method to derive a new family of criteria based on generic linear functionals. We then specialize this method to derive witnesses based on $s$-parametrized quasiprobability functions, generalizing previous criteria based on the Wigner function. In particular, we discuss in detail and analyze the properties of Husimi $Q$-function-based witnesses and prove that they are often more effective than previous criteria in detecting quantum non-Gaussianity of various kinds of non-Gaussian states evolving in a lossy channel.
\end{abstract}

DOI: 10.1103/PhysRevA.90.013810

PACS number(s): 42.50.Dv, 03.67.-a

\section{INTRODUCTION}

The classification of quantum states of the harmonic oscillator according to classical vs nonclassical and Gaussian vs non-Gaussian paradigms has been an ongoing focus of research in quantum information for some time now. A number of criteria for the detection of nonclassicality have been introduced, based on phase-space distributions [1-12], ordered moments [13-15], and information-theoretic arguments [16-22]. Particular attention has been devoted both in characterizing the set of states with positive Wigner function [23-27] and in distinguishing Gaussian and non-Gaussian states [28-30]. The different measures of non-Gaussianity proposed have been, for example, used to characterize experimentally generated non-Gaussian states [31-33], but they could not discriminate between states that can be written as mixtures of Gaussian states and the so-defined quantum non-Gaussian states.

From a physical point of view this is a particularly important distinction, as quantum non-Gaussian states can be produced only by means of highly nonlinear processes, while states belonging to the Gaussian convex hull can be generated by means of Gaussian operations only and classical randomization. In $[35,36]$ the first attempt to detect quantum non-Gaussianity was pursued, by deriving witnesses based, respectively, on photon-number probabilities and on the Wigner function. The criterion [35] has been already used to detect quantum non-Gaussian states produced in different experimental settings [37-39].

We here present a framework to derive quantum nonGaussianity (QNG) witnesses based on generic linear functionals. We apply these results to the case of $s$-parametrized quasiprobability distributions, generalizing the criteria obtained in [36] for the Wigner function, to the Husimi $Q$ function $(s=-1)$ and in general to any distribution characterized by a parameter $s<0$.

The paper is structured as follows. In Sec. II we present the problem by defining QNG, while Sec. III illustrates how to derive bounds of linear functionals on the Gaussian convex hull, along with their most important properties. In Sec. IV we present sufficient but not necessary QNG witnesses based on quasiprobability distributions. In Sec. V the effectiveness of these criteria is investigated for Fock states, photon-added coherent states, and photon-subtracted squeezed states, focusing in particular on the performances corresponding to the different quasiprobability distributions considered. In Sec. VI we illustrate how the uncertainty on the measured average photon number propagates to the derived bounds for different values of the parameter $s$. Section VII concludes the paper with final discussions and remarks.

\section{QUANTUM NON-GAUSSIANITY}

We begin by recalling the definition of the Gaussian convex hull

$$
\left.\mathcal{G}=\left\{\rho \in \mathcal{B}(\mathcal{H})\left|\rho=\int d \lambda p(\lambda)\right| \psi_{G}(\lambda)\right\rangle\left\langle\psi_{G}(\lambda)\right|\right\},
$$

where $p(\lambda)$ can be an arbitrary probability distribution, $\left|\psi_{G}(\boldsymbol{\lambda})\right\rangle$ are pure Gaussian states, and $\mathcal{B}(\mathcal{H})$ is the set of bounded operators. In general, all pure single-mode Gaussian states can be parametrized as $\left|\psi_{G}(\lambda)\right\rangle=D(\alpha) S(\xi)|0\rangle$, where $D(\alpha)$ and $S(\xi)$ are, respectively, the displacement and squeezing operators with the standard form presented in [34], $|0\rangle$ is the vacuum state, $\alpha, \xi$ are arbitrary complex numbers, and $\lambda=\{\alpha, \xi\}$. The set $\mathcal{G}$ includes mixed Gaussian states as they can always be decomposed in the form (1), but also non-Gaussian states, that is, states having a non-Gaussian Wigner function, as mixtures of coherent and squeezed states.

In line with Refs. [35,36], a quantum state $\rho$ is defined quantum non-Gaussian if and only if $\rho \notin \mathcal{G}$. To understand the importance of QNG in quantum optics, consider a simple example: Given a single-mode field initially prepared in the vacuum state, it is easy to verify that states belonging to $\mathcal{G}$ can be prepared by applying a combination of Gaussian operations and classical randomization. In contrast, the preparation of a quantum non-Gaussian state $\rho \notin \mathcal{G}$ starting from the vacuum field can only be achieved by means of some non-Gaussian operation, such as the application of a highly nonlinear Hamiltonian (i.e., more than quadratic in the mode operators) or probabilistic non-Gaussian operations such as photon addition or subtraction [40]. 


\section{BOUNDING LINEAR FUNCTIONALS ON THE GAUSSIAN CONVEX HULL}

Before proceeding to specialize our analysis to phase-space quasiprobability distributions, it is worthwhile to discuss the general approach we shall take in order to witness QNG. Suppose that a single-oscillator quantum state $\varrho$ is the output of some experiment. Assume that the data of our experiment allows us to estimate a certain quantity $\Phi[\varrho]$, where $\Phi$ is a linear functional on the space of quantum states, and a bound $n$ on the average photon number, that is $\operatorname{Tr}\left[\varrho \hat{a}^{\dagger} \hat{a}\right] \leqslant n$. Remarkably, it may be possible to gain some information on the QNG character of the state $\varrho$, solely based on those two quantities. To see this, let us consider

$$
\mathcal{G}_{n} \equiv\left\{\varrho_{G} \in \mathcal{G} \mid \operatorname{Tr}\left[\varrho_{G} \hat{a}^{\dagger} \hat{a}\right] \leqslant n\right\},
$$

which can be easily seen to be convex subsets of $\mathcal{G}$ for any $n \geqslant 0$, and define the function

$$
B(n) \equiv \min _{\varrho_{G} \in \mathcal{G}_{n}} \Phi\left[\varrho_{G}\right] .
$$

In other words, $B(n)$ is the lowest possible value that $\Phi[\varrho]$ could take compatible with the assumptions (i) $\varrho \in \mathcal{G}$ and (ii) $\operatorname{Tr}\left[\varrho \hat{a}^{\dagger} \hat{a}\right] \leqslant n$. Hence, if our state verifies (ii), but we find the quantity $\Phi[\varrho]$ to be below $B(n)$, we must conclude that $\varrho \notin \mathcal{G}$ [conversely, finding $\Phi[\varrho] \geqslant B(n)$ must be interpreted as an inconclusive result].

A key step in this procedure is the calculation of the function $B(n)$ for a given $\Phi$. In general, this can be seen as a problem of linear optimization over an infinite-dimensional parameter space [see Eq. (3)]. Luckily, the optimization can be dramatically simplified by exploiting the properties of $B(n)$. It turns out that it is sufficient to look for the (constrained) minimum of $\Phi$ amongst the set of pure Gaussian states and that of rank-2 mixtures of Gaussian states. Therefore, for a fixed $\Phi$ and $n$, only a finite number of parameters needs to be optimized in order to find $B(n)$. While one may be able to derive these results by applying standard techniques of convex analysis, we find it worthwhile to present their proof in our context in Appendix A.

\section{QUANTUM NON-GAUSSIANITY WITNESSES IN PHASE SPACE}

In the present work we show that that the structure of the states in Eq. (1) implies nontrivial constraints on their associated quasiprobability distributions. As a consequence, we will be able to certify QNG when those constraints are violated. We start by recalling the results of [36], where it was shown that the Wigner function of any quantum state $\varrho$ belonging to the Gaussian convex hull satisfies the following inequality:

$$
W[\varrho](0) \geqslant \frac{2}{\pi} \exp \{-2 n(1+n)\}, \quad n=\operatorname{Tr}\left[\varrho a^{\dagger} a\right] .
$$

We aim at obtaining bounds for other $s$-parametrized quasiprobabilities, which we express as a convolution [41]. For a quantum state of density operator $\varrho$,

$$
Q_{s}[\varrho](\alpha)=\frac{1}{\pi^{2}} \int d^{2} \xi \chi[\varrho](\xi, s) e^{\alpha \xi^{*}-\alpha^{*} \xi} .
$$

Here $\chi[\varrho](\xi, s)$ is the $s$-ordered characteristic function

$$
\chi[\varrho](\xi, s)=\operatorname{Tr}[\varrho \hat{D}(\xi)] e^{s|\xi|^{2} / 2} .
$$

There are three values of $s$ for which the quasiprobability function is typically explored: $s=1$ is the Glauber-Sudarshan $P$ function [2,3], $s=0$ is the Wigner function [1], and $s=-1$ is the Husimi $Q$ function [42]. For the purposes of this paper, the only necessary requirement on the parameter $s$ is going to be $s<0$, in order to avoid singularities in our quasiprobability distributions. Even though the function in Eq. (5) may lose some of the appealing properties of a quasiprobability distribution when $s<-1$, it still allows us to obtain useful and experimentally friendly QNG criteria, as we discuss in Sec. V.

\section{A. General QNG criteria in phase space}

The general problem under investigation can be formulated in the general framework of Sec. III, by noting that $Q_{s}[\varrho](\alpha)$ is a linear functional of the state at fixed $\alpha$ and $s$. Thus, having fixed a particular value of $s<0$, and assuming $\operatorname{Tr}\left[\varrho a^{\dagger} a\right] \leqslant n$, we ask ourselves whether the structure given in Eq. (1) implies a nontrivial lower bound on the possible values that $Q_{s}$ can take. More precisely, we define

$$
B_{s}(n) \equiv \min _{\varrho \in \mathcal{G}_{n}} Q_{s}[\varrho](0) .
$$

For every value of $s<0, B_{s}(n)$ is positive and convex. Moreover, we show in Appendix B that $B_{s}(n)$ is strictly decreasing in $n, B_{s}(n) \rightarrow 0$ as $n \rightarrow \infty$, and the minimizing state in $\mathcal{G}_{n}$ has an average photon number exactly equal to $n$. The functions $B_{s}$ are therefore nontrivial and can be exploited in the formulation of QNG criteria as follows.

Criterion 1. For a quantum state $\varrho$, define the QNG witness

$$
\Delta_{s}^{(a)}[\varrho]=Q_{s}[\varrho](0)-B_{s}(\bar{n}),
$$

where $\operatorname{Tr}\left[\varrho a^{\dagger} a\right] \leqslant \bar{n}$. Then

$$
\Delta_{s}^{(a)}[\varrho]<0 \Longrightarrow \varrho \notin \mathcal{G}
$$

that is, $\varrho$ is quantum non-Gaussian.

Criterion 2. Consider now a quantum state $\varrho$ and a Gaussian map $\mathcal{E}_{G}$, or a convex mixture of such maps. Define

$$
\Delta_{s}^{(b)}\left[\varrho, \mathcal{E}_{G}\right]=Q_{s}\left[\mathcal{E}_{G}(\varrho)\right](0)-B_{s}\left(\bar{n}_{\mathcal{E}}\right),
$$

where $\operatorname{Tr}\left[\mathcal{E}_{G}(\varrho) a^{\dagger} a\right] \leqslant \bar{n}_{\mathcal{E}}$. Then

$$
\exists \mathcal{E}_{G} \text { such that } \Delta_{s}^{(b)}\left[\varrho, \mathcal{E}_{G}\right]<0 \Longrightarrow \varrho \notin \mathcal{G} \text {. }
$$

The proof of Eq. (11) is the same as that for Eq. (9) except that a Gaussian map $\mathcal{E}_{G}$ has now first been applied to the state. This results in a change in the mean photon number, but does not impact the procedure. A full proof is provided in [36]. Before proceeding further, we note that the monotonicity of $B_{s}$ implies that the criteria become harder to satisfy as $\bar{n}$ and $\bar{n}_{\mathcal{E}}$ are increased (indeed, both $\Delta_{s}^{(a)}$ and $\Delta_{s}^{(b)}$ would correspondingly increase). Therefore, in the remainder of this paper we apply these witnesses respectively for $\operatorname{Tr}\left[\varrho a^{\dagger} a\right]=\bar{n}$ and $\operatorname{Tr}\left[\mathcal{E}_{G}(\varrho) a^{\dagger} a\right]=\bar{n}_{\mathcal{E}}$, which provide the highest chance of detecting QNG. On the other hand, experimentally it may be more practical to estimate an upper bound to the average photon number, rather than its actual value. It is important 
to note that these criteria provide sufficient but not necessary bounds.

\section{B. Near-optimality of pure states}

As discussed in Sec. III, we can restrict the optimization in Eq. (7) to rank-1 and rank-2 mixtures of Gaussian states. In all the considered examples, however, we found strong numerical evidence that the minimum was being reached by a pure Gaussian state. We have thus proven the near optimality of pure Gaussian states for a number of $s$ values of interest through a semianalytical approach, whose details are provided in Appendix C. A pure-state lower bound to each quasiprobability can be defined as

$$
B_{s}^{P}(n) \equiv \min _{\left|\psi_{G}\right\rangle}\left\{Q_{s}\left[\left|\psi_{G}\right\rangle\left\langle\psi_{G}\right|\right](0) \mid\left\langle\psi_{G}\left|a^{\dagger} a\right| \psi_{G}\right\rangle \leqslant n\right\},
$$

where the $\left|\psi_{G}\right\rangle$ 's are pure Gaussian states. Clearly, the bound in Eq. (12) is, in practice, easier to calculate than the one in Eq. (7); however, $B_{s}^{P}(n) \geqslant B_{s}(n)$, since we cannot exclude that the minimum may be reached by a rank-2 state. Nevertheless, our numerical studies for the cases $s=$ $\{-1 / 4,-1 / 2,-1,-2,-3\}$ provide the bound

$$
\left|B_{s}^{P}(n)-B_{s}(n)\right| \lesssim 10^{-15} n,
$$

meaning that the pure state lower bound $B_{s}^{P}$ is an excellent approximation to the true bound $B_{s}(n)$ in a wide range of average photon numbers (see Appendix C). Direct calculations relating to this bound are shown in more detail in Appendix D. The level of approximation provided by Eq. (13) is sufficient to guarantee the validity of our findings in the following section.

\section{DETECTING QUANTUM NON-GAUSSIANITY OF STATES EVOLVING IN A LOSSY CHANNEL}

In this section we test the effectiveness of the criteria introduced in Sec. IV A. Specifically, we investigate whether these criteria can be exploited to certify that pure nonGaussian states evolving in a lossy channel remain quantum non-Gaussian. We consider initially pure non-Gaussian states evolving in a lossy bosonic channel described by the following master equation:

$$
\dot{\varrho}=\frac{\gamma}{2}\left(\hat{a} \varrho \hat{a}^{\dagger}-\hat{a}^{\dagger} \hat{a} \varrho\right)+\text { H.c. }
$$

The corresponding quantum channel $\mathcal{E}_{\epsilon}$ is Gaussian and can be characterized by a single parameter, $\epsilon=1-e^{-\gamma t}$. We look for the maximum values of $\epsilon$ such that the criteria are violated; in particular we define

$$
\begin{gathered}
\epsilon_{s}^{(a)}[\varrho]=\max \left\{\epsilon: \Delta_{s}^{(a)}\left[\mathcal{E}_{\epsilon}(\varrho)\right] \leqslant 0\right\} \\
\epsilon_{s}^{(b)}[\varrho]=\max \left\{\epsilon: \exists \mathcal{E}_{\mathrm{G}} \text { such that } \Delta_{s}^{(b)}\left[\mathcal{E}_{\epsilon}(\varrho), \mathcal{E}_{\mathrm{G}}\right] \leqslant 0\right\} .
\end{gathered}
$$

Since for $\epsilon>\frac{1}{2}$ no negativity of the Wigner function can be observed, we are interested in larger values of $\epsilon_{s}^{(a)}$ and $\epsilon_{s}^{(b)}$, so that our criteria are able to detect quantum non-Gaussian states with positive Wigner function. The usefulness of the Wigner-function-based criterion has been extensively shown in $[36,43]$. We start by comparing the witnesses $\Delta_{s}^{(a)}$ for initial Fock states, while next we will discuss both the witnesses $\Delta_{s}^{(a)}$ and $\Delta_{s}^{(b)}$ for initial photon-added coherent states and photon-subtracted squeezed states. For $\Delta_{s}^{(a)}$ we compare across three values of the parameter $s$, the special cases $s=0$ and $s=-1$ corresponding, respectively, to the Wigner and Husimi- $Q$ functions, and adding a third case at $s=-2$. The quasiprobability distributions in the origin of phase space can be evaluated as

$$
Q_{s}[\varrho](0)=\frac{2}{\pi(1-s)} \sum_{m}(-1)^{m}\left(\frac{1+s}{1-s}\right)^{m}\langle m|\varrho| m\rangle .
$$

It depends only on the photon-number probabilities $p_{m}=$ $\langle m|\varrho| m\rangle$ and thus can be, in principle, experimentally measured by means of photon-number resolving detectors. More in particular the Wigner function in the origin corresponds to the average value of the parity operator $\Pi=(-)^{a^{\dagger} a}$, while for $s \rightarrow-1$, i.e., for the Husimi $Q$ function, we have the projection over the vacuum state $Q_{-1}[\varrho](0)=\langle 0|\varrho| 0\rangle$, which is measurable both by means of on-off and photon-number resolving detectors or through heterodyne detection. If we rather consider values of $s<-1$, it is possible to prove that $Q_{s}[\varrho](\alpha)$ corresponds to the rescaled heterodyne probability distribution, obtained by means of detectors with efficiency $\eta=2 /(1-s)$, such that for $s=-2$ we have $\eta=2 / 3$ [44]. While the parameter $\epsilon$ characterizing the lossy channel is supposed to be unknown, and our goal is to understand the maximum value of noise such that our criteria will be able to detect quantum non-Gaussian states, the inefficiency of the detector is known to the experimentalist as it is possible to determine its value by probing the detector with known states. In fact, as illustrated in Fig. 1, considering different values of $s$ is equivalent to detecting QNG of unknown states evolved through a lossy channel with a choice of detectors, one corresponding to each $s$.

As regards the examination of $\Delta_{s}^{(b)}$, we focus on the special cases of $s=0$ and $s=-1$. In both cases we observe how, in particular in the low-energy regime, the witnesses derived for lower values of $s$ show a larger robustness against loss.

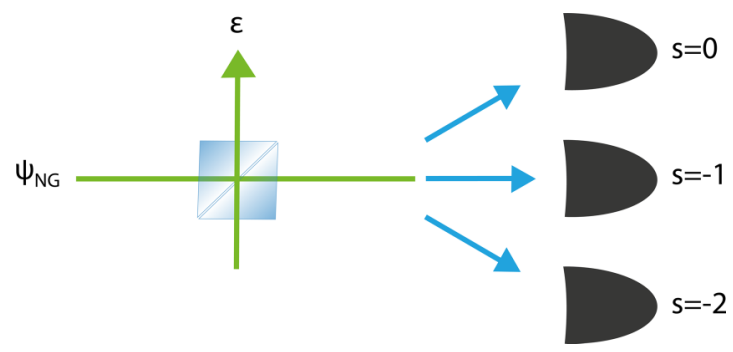

FIG. 1. (Color online) We send a non-Gaussian state through a channel with loss $\epsilon$ and choose the detector with which to measure it. The $s=0$ detector corresponds to parity measurement, $s=-1$ corresponds to the probability of vacuum detection, while $s=-2$ corresponds to an inefficient vacuum detection with efficiency $\eta=2 / 3$. 


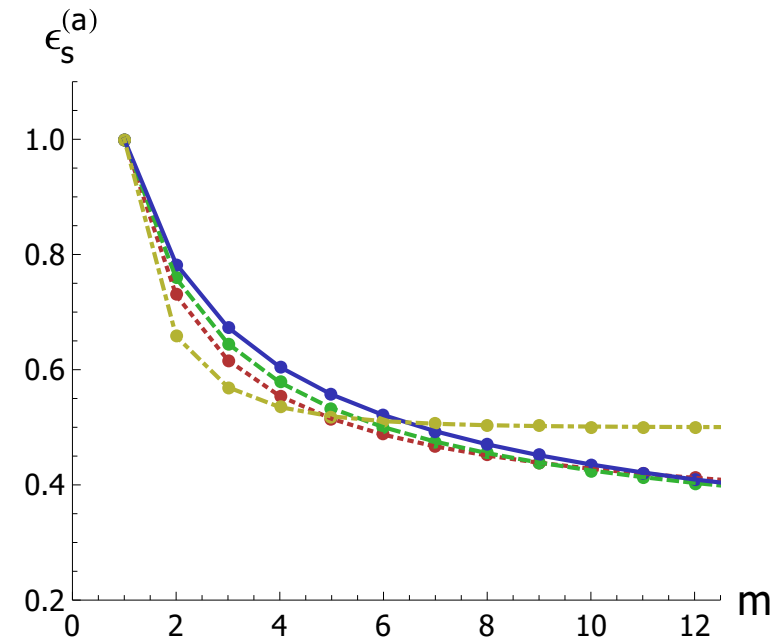

FIG. 2. (Color online) Maximum value of the loss parameter $\epsilon_{s}^{(a)}$ such that the bounds are violated, as a function of the initial Fock number $m$ and for different values of $s$ : yellow (dot-dashed line), $s=0 ;$ red (dotted line), $s=-1 / 2$; green (dashed line), $s=-1$; blue (solid line), $s=-2$.

\section{A. Fock states}

A Fock state $|m\rangle$ evolves in a lossy channel as

$$
\mathcal{E}_{\epsilon}(|m\rangle\langle m|)=\sum_{l=0}^{m}\left(\begin{array}{c}
m \\
l
\end{array}\right)(1-\epsilon)^{l} \epsilon^{m-l}|l\rangle\langle l|,
$$

and the value of the corresponding $s$-parametrized quasiprobability distribution at the origin can be evaluated using the formula for a generic Fock state

$$
Q_{s}[|m\rangle\langle m|](0)=\frac{2}{\pi(1-s)}(-1)^{m}\left(\frac{1+s}{1-s}\right)^{m} .
$$

We have evaluated the corresponding values of the witnesses $\Delta_{s}^{(a)}$, along with the maximum values of the noise parameter $\epsilon_{s}^{(a)}$ where the bounds are violated. In particular, these are plotted in Fig. 2 as a function of the Fock number $m$ and for different values of the $s$ parameter. Perhaps

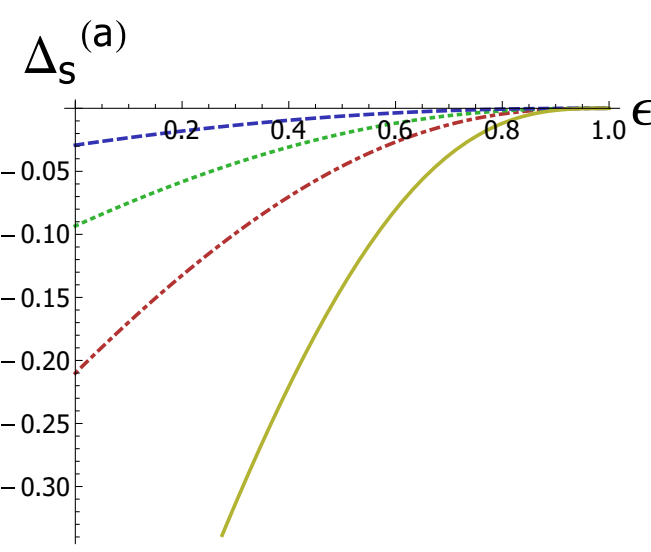

(a) surprisingly, the witnesses appear to be more sensitive as $s$ decreases; that is, they provide a larger value of $\epsilon_{s}^{(a)}$ in the relevant range $m \leqslant 5$.

One can notice an interesting tradeoff in the behavior of the witnesses $\Delta_{s}^{(a)}$ for the Fock state $|1\rangle$ in Fig. 3(a). We note that the absolute value of $\Delta_{s}^{(a)}$ is decreasing by considering more negative values of $s$. Even though such monotonous behavior is lost for the Fock state $|3\rangle$ [see Fig. 3(b)], similar conclusions can be drawn for higher Fock states. Hence, while one can, in principle, detect QNG for larger values of the noise parameter by decreasing $s$, the amount of violation quantified by $\Delta_{s}^{(a)}$ may be generally smaller. The impact of such tradeoff on the experimental detection of QNG, however, cannot be assessed without a thorough analysis of the propagation of experimental errors for the various witnesses. A first attempt towards this direction will be done in Sec. VI, while a complete analysis goes beyond the scope of our paper.

\section{B. Photon-added coherent states}

A photon-added coherent (PAC) state is defined as

$$
\left|\psi_{\mathrm{PAC}}\right\rangle=\mathcal{N} \hat{a}^{\dagger}|\alpha\rangle,
$$

where $\mathcal{N}$ is the normalization factor. Its average photon number is

$$
\bar{n}_{0}^{\mathrm{PAC}}=\frac{\alpha^{4}+3 \alpha^{2}+1}{1+\alpha^{2}},
$$

for $\alpha \in \mathbb{R}$. The $s$-parametrized quasiprobability distributions are determined using the convolution expression presented in [41]:

$$
Q_{s^{\prime}}[\varrho](\alpha)=\frac{2}{\pi\left(s-s^{\prime}\right)} \int d^{2} \beta Q_{s}[\varrho](\beta) e^{-\frac{2|\alpha-\beta|^{2}}{\left(s-s^{\prime}\right)}},
$$

with the condition that this holds provided $s^{\prime}<s$. Using this expression, the values of the witnesses $\Delta_{s}^{(a)}$ can be computed.

Similar to what was observed for the Fock states, smaller values of $s$ produce a more effective bound for the certification of QNG in noisy PAC, provided the parameter $\alpha$ is smaller or

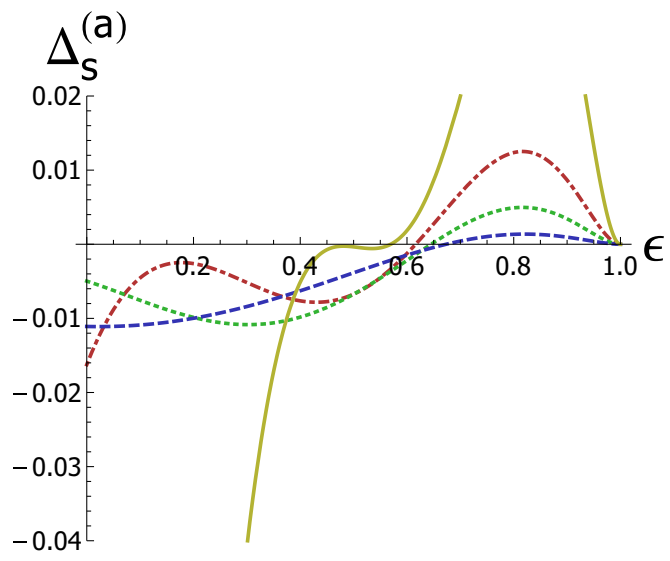

(b)

FIG. 3. (Color online) QNG witnesses $\Delta_{s}^{(a)}$ for initial Fock states (a) $|1\rangle$ and (b) $|3\rangle$ as a function of the lossy parameter $\epsilon$ and for different values of $s$ : yellow (solid line), $s=0$; red (dot-dashed line), $s=-1 / 2$; green (dotted line), $s=-1$; blue (dashed line), $s=-2$. 


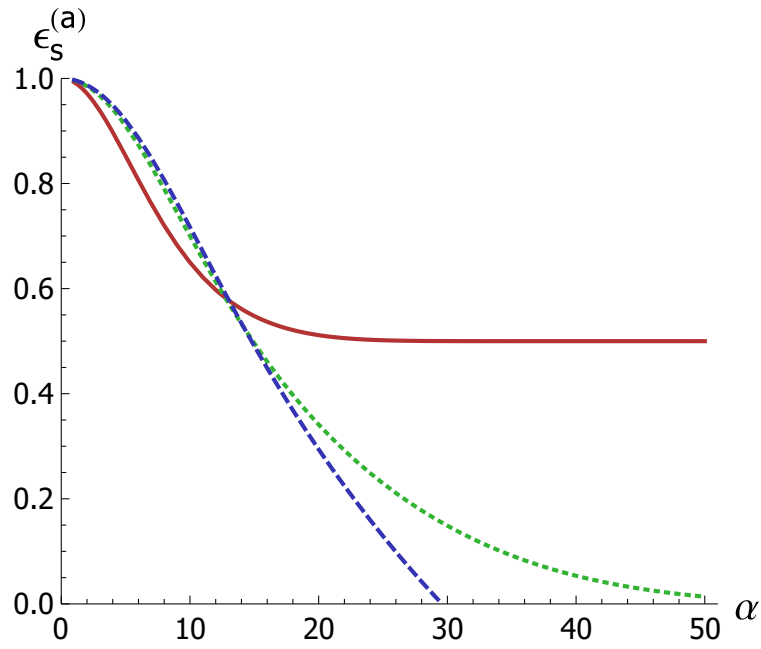

FIG. 4. (Color online) Maximum value of the loss parameter $\epsilon_{s}^{(a)}$ such that the bounds are violated, as a function of the coherent-state parameter $\alpha$ and for different values of $s$ : red (solid line), $s=0$; green (dotted line), $s=-1$; blue (dashed line), $s=-2$.

equal to about 10, as illustrated in Fig. 4. However, we observe again evidence that there is a compromise between a tighter bound and the amount of violation quantified by the criterion $\Delta_{s}^{(a)}$, with the magnitude of the parameter decreasing for lower values of $s$.

We can now consider the optimized witness defined in Eq. (10). For the PAC state, it is observed that the minima of the witness $\Delta_{s}^{(b)}[\varrho, D(\beta)]$ for the quasiprobability distributions are displaced from the origin of the phase space, and it is therefore possible to decrease the QNG indicator by redisplacing the minimum to the origin. Thus, the quasiprobability function $Q_{s}[\varrho](-\beta)$ and the average photon number of the displaced state are computed, yielding

$$
\bar{n}^{\mathrm{PAC}}(\beta)=(1-\epsilon)|\beta|^{2} \bar{n}_{0}^{\mathrm{PAC}}+\sqrt{1-\epsilon}\left(\beta^{*}\langle\hat{a}\rangle_{0}+\beta\left\langle\hat{a}^{\dagger}\right\rangle_{0}\right),
$$

where $\langle A\rangle_{0}=\left\langle\psi_{0}|A| \psi_{0}\right\rangle$, and for $\left|\psi_{0}\right\rangle=\left|\psi_{\mathrm{PAC}}\right\rangle$,

$$
\langle\hat{a}\rangle_{0}=\left\langle\hat{a}^{\dagger}\right\rangle_{0}=\frac{\alpha\left(2+\alpha^{2}\right)}{1+\alpha^{2}} .
$$

We then minimize $\Delta_{s}^{(b)}[\varrho, D(\beta)]$ over the possible displacement parameters $\beta$. We find that the optimal value of $\beta$ for large values of $\epsilon$ and $\alpha \geqslant 1.5$, which is nearly the same for the Wigner and $Q$ functions, can be approximated as

$$
\beta_{\mathrm{opt}} \simeq-\alpha \sqrt{1-\epsilon}=-\alpha e^{-\gamma t / 2} .
$$

Taking $\beta=\beta_{\mathrm{opt}}$, we compare the values of the QNG witness based on the second criterion for $s=0$ and $s=-1$. Both the plots and numerical investigations indicate that $\epsilon_{s}^{(b)} \simeq 1$ for all possible values of $\alpha$. No root can be found for general $\alpha$.

\section{Photon-subtracted squeezed states}

Taking the squeezing parameter $r$ to be real, define the photon-subtracted squeezed (PSS) state as $\left|\psi_{\text {PSS }}\right\rangle=$ $\mathcal{N} \hat{a} S(r)|0\rangle$. The average photon number for the PSS state is

$$
\bar{n}_{0}^{\mathrm{PSS}}=3 \sinh ^{2} r+1 .
$$

The quasiprobability distributions for the PSS state are computed using the convolution of Eq. (22). The bounds were found and again demonstrate the same characteristics found with the other states. In this case, more negative values of $s$ allow for a larger value of the loss parameter $\epsilon_{s}^{(a)}$ for squeezing parameter $r \lesssim 8$ [Fig. 5(a)]. Again, though, this represents a loss in the quantity of violation described by the parameter $\Delta_{s}^{(a)}$.

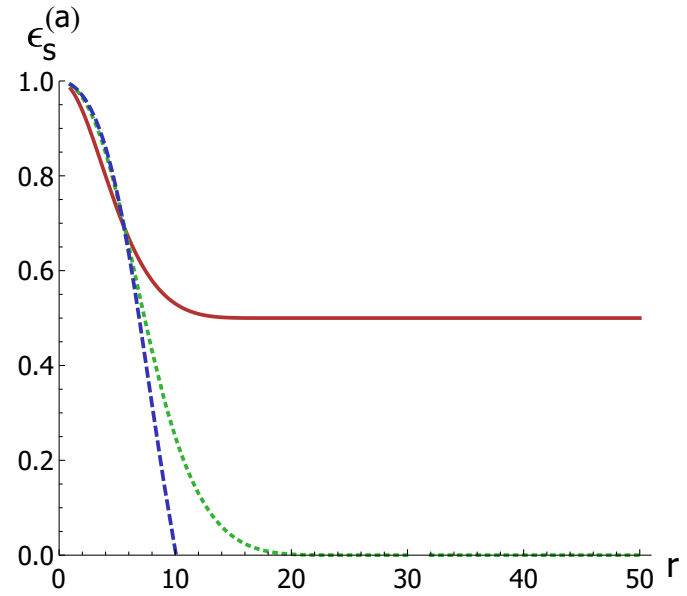

(a)

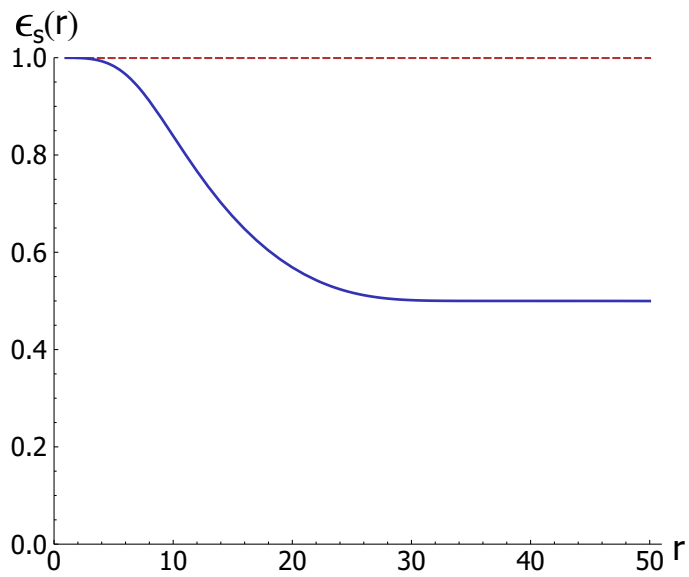

(b)

FIG. 5. (Color online) Loss parameters for PSS states. (a) Maximum value of the loss parameter $\epsilon_{s}^{(a)}$ such that the bounds are violated, as a function of the squeezing parameter $r$ and for different values of $s$ : red (solid line), $s=0$; green (dotted line), $s=-1$; blue (dashed line), $s=-2$. (b) A comparison between the maximum values of the noise parameter $\epsilon_{s}^{(b)}$ for PSS states by using the Wigner function criterion (blue solid line) and the $Q$-function criterion (red dashed line). 
Similar to how PAC states inherit a displacement, PSS states inherit additional squeezing on the evolved state, and we can use the optimized witness (10) by using additional squeezing operations. In this case, there is a difference between how the Wigner function changes under this squeezing versus how the $Q$ function changes: While the Wigner function at the origin is unchanged by the squeezing operation, the $Q$ function is not invariant and as a result the following argument for the optimization of the squeezing parameter is only valid for the Wigner function. This proves not to be a problem and will be discussed below. First, we determine the value $q_{\text {opt }}$ that minimizes the average photon number of $S(q) \rho S^{\dagger}(q)$,

$$
\begin{aligned}
\bar{n}^{\mathrm{PSS}}(q)= & (1-\epsilon)\left[\bar{n}_{0}^{\mathrm{PSS}}\left(\mu_{q}^{2}+v_{q}^{2}\right)\right. \\
& \left.+\mu_{q} v_{q}\left(\left\langle\hat{a}^{2}\right\rangle_{0}+\left\langle\hat{a}^{\dagger 2}\right\rangle_{0}\right)\right]+v_{q}^{2},
\end{aligned}
$$

where $\mu_{t}=\cosh t, v_{t}=\sinh t$, and, for an initial PSS state $\left|\psi_{\mathrm{PSS}}\right\rangle,\left\langle\hat{a}^{2}\right\rangle_{0}=\left\langle\hat{a}^{\dagger 2}\right\rangle_{0}=3 \mu_{r} v_{r}$. In this case the optimal squeezing value can be evaluated analytically:

$$
\begin{gathered}
q_{\mathrm{opt}}=-\operatorname{arccosh}\left(\mu_{\mathrm{opt}}\right) \\
\mu_{\mathrm{opt}}=\frac{1}{\sqrt{2}}\left[1+\frac{6(1-\epsilon) \mu_{r}^{2}+4 \epsilon-3}{\sqrt{(4 \epsilon-3)^{2}+12(1-\epsilon) \epsilon \mu_{r}^{2}}}\right]^{1 / 2} .
\end{gathered}
$$

We again follow the format of the PAC-state analysis, assigning the squeezing parameter its optimal value $q_{\text {opt }}$ and plotting the criterion as a function of $\epsilon$. We plot both the Wigner and $Q$ functions for $q_{\text {opt }}$ and illustrate that while the $q_{\text {opt }}$ used is only optimized for the Wigner function, the maximum noise $\epsilon_{s}^{(b)}$ for the $Q$ function for this value of $s$ is 1 for all values and therefore already giving the desired result. So even if this is not the optimal squeezing for the $Q$ function, it is sufficient to detect QNG by means of the $Q$ function-based witness. This feature is illustrated in Fig. 5(b).

\section{ESTIMATION OF ERROR ON THE BOUNDS}

While a full error propagation to evaluate the various witnesses is beyond the scope of this paper, it is straightforward to evaluate the bounds $B_{s}(n)$ for the different $s$ values, based on uncertainty in the mean photon number $n$. The method of determining the error on the bound is chosen to best approximate the experimental realities. To this end, we suppose that we have a photon-number resolving detector with which we would like to measure different average values of $n$ that we assign to a set $n_{\text {avg. These values are a discretized }}$ version of the range of $n$ we would like to consider. In an experiment, we need to measure our state $k$ times, for preferably large $k$. We then define $n_{\text {tot }}$ as the total number of photons measured over all $k$ trials; that is,

$$
n_{\mathrm{tot}}=k \times n_{\mathrm{avg}} \text {. }
$$

For $n_{\text {tot }}$ we assume a Poissonian distribution for simplicity. Before evaluating the means and variances of the bounds we divide by $k$ as we wish to evaluate for a distribution about $n_{\text {avg. Normalizing the results so all bounds evaluate to } 1 \text { at }}$ $n_{\text {avg }}=0$, we get the distributions in Fig. 6. As we can observe, the errors on the different bounds $B_{s}(n)$ are comparable. This shows how the errors coming from the measurement of the

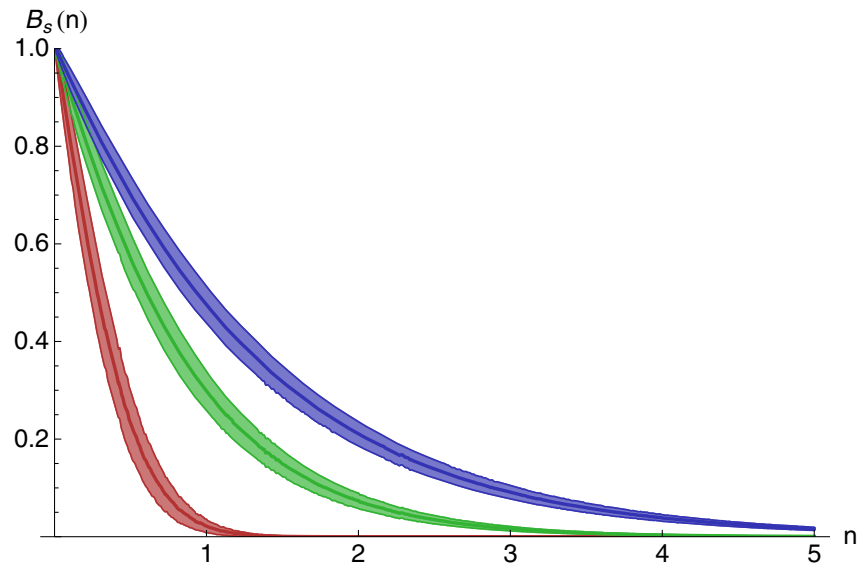

FIG. 6. (Color online) Error on the (renormalized) bounding functions $B_{s}(n)$ for (from top to bottom) $s=0$ (blue line), $s=-1$ (green line), and $s=-2$ (red line).

quasiprobability distributions values $Q_{s}[\varrho](\alpha)$, will probably play a major role when the proposed witnesses will be used in an actual experiment.

\section{CONCLUSIONS}

We have presented a general method to derive bounds of linear functionals on the Gaussian convex hull. We note that these bounds are sufficient but not necessary for the characterization of this set. After having presented the main properties of the bounds, we used it to define QNG witnesses based on $s$-parametrized quasiprobability distributions, with $s<0$. The witnesses are based on bounding from above the average photon number of the quantum state, and measuring the value of the corresponding quasiprobability distribution in a particular point of phase space (typically the origin).

Following the determination of these witnesses, we consider three different states and test the criteria for three different values of $s$ for each state. Motivation to consider the bound for different $s$ values comes from the freedom it provides to change the type of detection used in experiment. While it is known that $s=0$ and -1 correspond to the Wigner and $Q$ functions, respectively, $s=-2$ is comparable to measuring the $Q$ function with an inefficient detector. As the inefficiency of the detector can be known from trials using known states, allowing for $s<-1$ provides a more general description less dependent on the type of detection. From the different states for which the bound was considered, we see that there is a region for which a smaller $s$ value provides a witness for QNG and allows more channel loss than the originally considered Wigner function bound. There is, however, a tradeoff between the maximum amount of loss for which QNG may be witnessed and the amount of violation quantified by the criterion which is generally smaller, for smaller values of $s$.

\section{ACKNOWLEDGMENTS}

We thank M. Barbieri, D. Dorigoni, S. Olivares, and R. Filip for fruitful discussions. M.G.G. acknowledges support from EPSRC (Grant No. EP/K026267/1). T.T. and M.S.K. acknowledge support from Grant No. NPRP 4-554-1-084 from Qatar National Research Fund. M.G.A.P. acknowledges support 
from MIUR (Grant No. FIRB-LiCHIS- RBFR10YQ3H). M.S.K. also thanks the UK EPSRC for support.

\section{APPENDIX A: GENERAL PROPERTIES OF THE BOUNDS}

In this section we prove that if the minimum of $\Phi$ on $\mathcal{G}_{n}$ is not achieved by a pure Gaussian state, then it must be achieved by a rank- 2 mixture of pure Gaussian states. Before proving this, it is useful to introduce an auxiliary lemma.

Lemma 1. The function $B(n)$ is convex.

Proof. Let $\rho_{1} \in \mathcal{G}_{n_{1}}$ and $\rho_{2} \in \mathcal{G}_{n_{2}}$ be such that $B\left(n_{1}\right)=$ $\Phi\left[\rho_{1}\right]$ and $B\left(n_{2}\right)=\Phi\left[\rho_{2}\right]$, and take $0 \leqslant p \leqslant 1$. Then

$$
\begin{array}{rl}
p & B\left(n_{1}\right)+(1-p) B\left(n_{2}\right) \\
& =p \Phi\left[\rho_{1}\right]+(1-p) \Phi\left[\rho_{2}\right] \\
& =\Phi\left[p \rho_{1}+(1-p) \rho_{2}\right] \geqslant B\left[p n_{1}+(1-p) n_{2}\right],
\end{array}
$$

where we have used the linearity of $\Phi$ and the fact that $p \rho_{1}+(1-p) \rho_{2} \in \mathcal{G}_{p n_{1}+(1-p) n_{2}}$ might not be the state which minimizes $\Phi$ in that set.

Theorem 1. Given $n$, either there exists a pure Gaussian state $\left|\psi_{n}\right\rangle$ of $\mathcal{G}_{n}$ such that $B(n)=\Phi\left[\left|\psi_{n}\right\rangle\left\langle\psi_{n}\right|\right]$, or $B(n)=\Phi\left[\rho_{n}\right]$, where $\rho_{n} \in \mathcal{G}_{n}$ is a rank-2 state of the form $\rho_{n}=p\left|\psi_{1}\right\rangle\left\langle\psi_{1}\right|+$ $(1-p)\left|\psi_{2}\right\rangle\left\langle\psi_{2}|,| \psi_{1}\right\rangle,\left|\psi_{2}\right\rangle$ being pure Gaussian states.

Proof. It is sufficient to consider mixtures comprising a finite number of pure Gaussian states. Infinite sums and integrals [such as that appearing in the definition (1)] are included in the discussion via a limiting procedure, thanks to the continuity of $\Phi$. Suppose $B(n)=\Phi\left(\rho_{n}\right), \rho_{n}=\sum_{j} p_{j}\left|\psi_{j}\right\rangle\left\langle\psi_{j}\right|$, and the $\left|\psi_{j}\right\rangle$ 's are pure Gaussian states of average photon number $n_{j}$, such that $\bar{n} \equiv \sum p_{j} n_{j} \leqslant n$. From Eq. (3) and the fact that $\mathcal{G}_{n} \subset \mathcal{G}_{m}$ for $m>n$, it follows that $B(n)$ is a nonincreasing function of $n$ (that is, the minimum is, in general, lower on a larger set). Then

$$
\begin{aligned}
B(\bar{n}) \geqslant B(n) & =\Phi\left[\rho_{n}\right]=\Phi\left[\sum_{j} p_{j}\left|\psi_{j}\right\rangle\left\langle\psi_{j}\right|\right] \\
& =\sum_{j} p_{j} \Phi\left[\left|\psi_{j}\right\rangle\left\langle\psi_{j}\right|\right] \geqslant \sum_{j} p_{j} B\left(n_{j}\right) \geqslant B(\bar{n}),
\end{aligned}
$$

where the first inequality follows from the nonincreasing behavior of $B$, the second inequality follows from the fact that $\left|\psi_{j}\right\rangle \in \mathcal{G}_{n_{j}}$ may not be the state minimizing $\Phi$ on $\mathcal{G}_{n_{j}}$, and the third inequality follows from the convexity of $B$ proven in Lemma 1. To avoid contradiction, only the equal signs are possible in Eq. (A2). This also implies that for all pure states $\left|\psi_{j}\right\rangle$ involved in the sum it must be

$$
\Phi\left[\left|\psi_{j}\right\rangle\left\langle\psi_{j}\right|\right]=B\left(n_{j}\right) .
$$

If $n_{J}=n$ for some $J$, then $B(n)=\Phi\left[\left|\psi_{J}\right\rangle\left\langle\psi_{J}\right|\right]$.

Otherwise, there must be at least two values $j_{1}$ and $j_{2}$ in the sum such that $n_{j_{1}}<\bar{n}<n_{j_{2}}$; thus, one can find $0<q<1$ yielding $q n_{j_{1}}+(1-q) n_{j_{2}}=\bar{n}$. For $r$ sufficiently small (and positive), it is possible to decompose

$$
\begin{aligned}
\sum p_{j}\left|\psi_{j}\right\rangle\left\langle\psi_{j}\right|= & r\left[q\left|\psi_{j_{1}}\right\rangle\left\langle\psi_{j_{1}}|+(1-q)| \psi_{j_{2}}\right\rangle\left\langle\psi_{j_{2}}\right|\right] \\
& +(1-r) \sum \tilde{p}_{j}\left|\psi_{j}\right\rangle\left\langle\psi_{j}\right|
\end{aligned}
$$

where $\left\{\tilde{p}_{j}\right\}$ is a probability distribution such that $\sum \tilde{p}_{j} n_{j}=\bar{n}$. Correspondingly, Eq. (A2) implies

$$
\begin{aligned}
B(\bar{n})= & r\left\{q \Phi\left[\left|\psi_{j_{1}}\right\rangle\left\langle\psi_{j_{1}}\right|\right]+(1-q) \Phi\left[\left|\psi_{j_{2}}\right\rangle\left\langle\psi_{j_{2}}\right|\right]\right\} \\
& +(1-r) \sum \tilde{p}_{j} \Phi\left[\left|\psi_{j}\right\rangle\left\langle\psi_{j}\right|\right] \\
= & r\left[q B\left(n_{j_{1}}\right)+(1-q) B\left(n_{j_{2}}\right)\right]+(1-r) \sum \tilde{p}_{j} B\left(n_{j}\right) \\
\geqslant & r B(\bar{n})+(1-r) B(\bar{n})=B(\bar{n}),
\end{aligned}
$$

where we have exploited Eq. (A3) and the convexity of $B$. This implies that it must be $q B\left(n_{j_{1}}\right)+(1-q) B\left(n_{j_{2}}\right)=B(\bar{n})$. Moreover, we had $B(n)=B(\bar{n})$. Therefore,

$$
B(n)=q \Phi\left[\left|\psi_{j_{1}}\right\rangle\left\langle\psi_{j_{1}}\right|\right]+(1-q) \Phi\left[\left|\psi_{j_{2}}\right\rangle\left\langle\psi_{j_{2}}\right|\right] .
$$

We have thus proven that $B(n)$ is either achieved by a pure Gaussian state or by a rank- 2 mixture of pure Gaussian states.

\section{APPENDIX B: PROPERTIES OF THE QUASIPROBABILITY BOUNDS}

For a single-mode pure Gaussian quantum state $\left|\psi_{G}\right\rangle=$ $D(\alpha) S(\xi)|0\rangle$, with $\alpha=|\alpha| e^{i \theta}, \xi=r e^{i \phi}$ the value of the $s$ parametrized quasiprobability distribution in the origin can be written as

$$
Q_{s}\left[\left|\psi_{G}\right\rangle\left\langle\psi_{G}\right|\right](0)=\frac{2 e^{-\frac{2(n-m)[1+2 m-2 \sqrt{m(1+m)} \cos (2 \theta-\phi)-s]}{1+s(s-2-4 m)}}}{\pi \sqrt{1+s(s-2-4 m)}},
$$

where $n=|\alpha|^{2}+\sinh ^{2} r$ is the average number of photons and $m=\sinh ^{2} r \leqslant n$ is the squeezing fraction. The condition $s<0$ ensures that the expression in Eq. (B1) is real. From this expression, we can prove some general properties of the functions $B_{s}(n)$. Firstly, we notice that $B_{s}(n)>0$ for any $n \geqslant$ 0 . Also, since the only state in $\mathcal{G}_{0}$ is the vacuum $|0\rangle$, we have

$$
B_{s}(0)=\frac{2}{\pi \sqrt{1+s(s-2)}} .
$$

One can also see that $\lim _{n \rightarrow \infty} B_{s}(n)=0$ for any $s<0$. Then, it is easy to show that $B_{s}(n)$ is strictly decreasing: suppose that $\tilde{n}>n$ but $B_{s}(\tilde{n})=B_{s}(n)$. Since $B_{s}$ tends to zero for large $n$, it is possible to find $N>\tilde{n}>n$ such that $B_{s}(N)<B_{s}(\widetilde{n})$ and $q \in(0,1)$ such that $q n+(1-q) N=\tilde{n}$. Then one would obtain $q B_{s}(n)+(1-q) B_{s}(N) \geqslant B_{s}(\tilde{n})=B_{s}(n)$; on the other hand, $q B_{s}(n)+(1-q) B_{s}(N)<q B_{s}(n)+(1-q) B_{s}(\tilde{n})=$ $B_{s}(n)$, thus reaching a contradiction.

Finally, we show that the bound $B_{s}(n)$ is achieved by a state with $n$ average photons, that is $B_{s}(n)=Q_{s}\left[\rho_{n}\right](0)$ and $\operatorname{Tr}\left[\rho_{n} a^{\dagger} a\right]=n$ [for brevity, in what follows we omit the phase-space argument of $Q_{s}$, assuming it to be always "(0)"]. Assuming that this is not the case, we write $B_{s}(n)=Q_{s}\left[\rho_{\tilde{n}}\right]$, such that $\operatorname{Tr}\left[\rho_{\widetilde{n}} a^{\dagger} a\right]=\tilde{n}<n$. However, one has $\rho_{\tilde{n}} \in \mathcal{G}_{\widetilde{n}} \subset$ $\mathcal{G}_{n}$, and as a consequence we reach the absurd conclusion $B_{s}(n)=Q_{s}\left[\rho_{\tilde{n}}\right] \geqslant B_{s}(\tilde{n})$, which is in contradiction with the strict monotonicity of $B_{s}$.

We remark that all the properties derived in this section hold for any linear functional whose bound satisfies the properties (i) $B(0)>0$ and (ii) $\lim _{n \rightarrow \infty} B(n)=0$. 


\section{APPENDIX C: NEAR OPTIMALITY OF PURE STATES}

We note that, in general, it must be $B_{s}^{P}(n) \geqslant B_{s}(n)$, since we cannot exclude that the minimum may be reached by a rank- 2 state. By adopting the same reasoning as in Ref. [36], however, one can show that if $B_{s}^{P}(n)$ is convex in the variable $n$, then it must be that $B_{s}^{P}(n)=B_{s}(n)$. While we have the conjecture that this is the case for any $s \leqslant 0$, the functional form of $B_{s}^{P}$ is, in general, too cumbersome to verify its convexity analytically. Adopting a numerical approach we have verified that, for the values $s=\{-1 / 4,-1 / 2,-1,-2,-3\}$, the function $B_{s}^{P}(n)$ is convex for $n \leqslant n_{\max } \simeq 10^{15}$ (that is, its second derivative is positive) [45].

Then, using the results of Appendix A we note that the only possibility to have $B_{s}^{P}(n) \neq B_{s}(n)$ is when $B_{s}(n)=(1-$ p) $B_{s}^{P}\left(n_{1}\right)+p B_{s}^{P}\left(n_{2}\right)$, with the average photon numbers of the two pure Gaussian states and the probability $p$ verifying, respectively, $n_{1}<n, n_{2}>n_{\max }$, and $p<n / n_{\max }$. Thus, we have

$$
\begin{aligned}
0 \leqslant & B_{s}^{P}(n)-B_{s}(n)=B_{s}^{P}(n)-B_{s}^{P}\left(n_{1}\right) \\
& +p\left[B_{s}^{P}\left(n_{1}\right)-B_{s}^{P}\left(n_{2}\right)\right] \leqslant p B_{s}^{P}\left(n_{1}\right) \\
< & \frac{2}{\pi \sqrt{1+s(s-2)}} \frac{n}{n_{\max }} \sim 10^{-15} n,
\end{aligned}
$$

where we have used $B_{s}^{P}(n)-B_{s}^{P}\left(n_{1}\right) \leqslant 0$, which follows from $n_{1}<n$ and $B_{s}^{P}(n)$ being monotonically decreasing in $n$ [this can be seen easily from the abstract definition in Eq. (12), or more directly from Eq. (D1)].

\section{APPENDIX D: PURE-STATE BOUNDS}

Our goal is now to minimize the function in Eq. (B1) over all pure Gaussian states with average photon number $n$. We first notice that setting $2 \theta-\phi=\pi$ yields the inequality

$$
Q_{s}\left[\left|\psi_{G}\right\rangle\left\langle\psi_{G}\right|\right](0) \geqslant \frac{2 e^{-\frac{2(n-m)[1+2 m+2 \sqrt{m(1+m)}-s]}{1+s(s-2-4 m)}}}{\pi \sqrt{1+s(s-2-4 m)}} .
$$

Finally, one has to minimize the above expression with respect to the squeezing fraction $m$, under the constraint $m \leqslant n$. The optimizing value of $m$ for a given $s$ is denoted $m_{s}(n)$. As an example, we here consider the case $s=-1$, where the function $Q_{-1}$ is the so-called Husimi $Q$ function

$$
Q_{-1}[\varrho](\alpha)=\frac{\langle\alpha|\varrho| \alpha\rangle}{\pi} .
$$

Here $|\alpha\rangle=D(\alpha)|0\rangle$ denotes a coherent state, showing that the Husimi $Q$ function corresponds to the heterodyne probability distribution of the quantum state $\varrho$. For a generic state $\varrho \in \mathcal{G}$, the value of the squeezed photon number for which the Husimi $Q$ function in the origin is minimized is

$$
\begin{aligned}
& m_{-1}(n)=\frac{1}{6}\{2(n-1)+\sqrt{3} \operatorname{Im}[g(n)]-\operatorname{Re}[g(n)]\}, \\
& \text { where } \quad \begin{aligned}
g(n)= & \left\{-17-21 n+3 n^{2}+8 n^{3}+3 i(1+n)\right. \\
& \times \sqrt{6+3 n[24+n(37+16 n)]}\}^{1 / 3} .
\end{aligned}
\end{aligned}
$$

The bound $B_{-1}^{P}(n)$ can be then obtained by substituting this function into the form of Eq. (D1) where $s=-1$,

$$
Q_{-1}\left[\left|\psi_{G}\right\rangle\left\langle\psi_{G}\right|\right](0) \geqslant \frac{2 e^{-\frac{2\left[n-m_{-1}(n)\left\{1+2 m_{-1}(n)+2 \sqrt{m_{-1}(n)\left[1+m_{-1}(n)\right]}+1\right\}\right.}{1+\left(3+4 m_{-1}(n)\right)}}}{\pi \sqrt{1+\left[3+4 m_{-1}(n)\right]}},
$$

Where the final result is too cumbersome to be reported here. Identical approaches can be effectively pursued for other values of $s<0$.
[1] E. Wigner, Phys. Rev. 40, 749 (1932).

[2] R. J. Glauber, Phys. Rev. 131, 2766 (1963).

[3] E. C. G. Sudarshan, Phys. Rev. Lett. 10, 277 (1963).

[4] C. T. Lee, Phys. Rev. A 44, R2775 (1991).

[5] C. T. Lee, Phys. Rev. A 45, 6586 (1992).

[6] Arvind, N. Mukunda, and R. Simon, Phys. Rev. A 56, 5042 (1997).

[7] Arvind, N. Mukunda, and R. Simon, J. Phys. A 31, 565 (1998).

[8] M. A. Marchiolli, V. S. Bagnato, Y. Guimaraes, and B. Baseia, Phys. Lett. A 279, 294 (2001).

[9] M. G. A. Paris, Phys. Lett. A 289, 167 (2001).

[10] V. V. Dodonov, J. Opt. B 4, R1 (2002).

[11] A. Kenfack and K. Zyczkowski, J. Opt. B 6, 396 (2004).

[12] J. Paavola, M. J. W. Hall, M. G. A. Paris, and S. Maniscalco, Phys. Rev. A 84, 012121 (2011).

[13] E. V. Shchukin and W. Vogel, Phys. Rev. A 72, 043808 (2005).

[14] T. Kiesel, W. Vogel, V. Parigi, A. Zavatta, and M. Bellini, Phys. Rev. A 78, 021804(R) (2008).

[15] W. Vogel, Phys. Rev. Lett. 100, 013605 (2008).

[16] R. Simon, Phys. Rev. Lett. 84, 2726 (2000).
[17] Lu-Ming Duan, G. Giedke, J. I. Cirac, and P. Zoller, Phys. Rev. Lett. 84, 2722 (2000).

[18] P. Marian, T. A. Marian, and H. Scutaru, Phys. Rev. Lett. 88, 153601 (2002).

[19] P. Giorda and M. G. A. Paris, Phys. Rev. Lett. 105, 020503 (2010); G. Adesso and A. Datta, ibid. 105, 030501 (2010).

[20] A. Ferraro and M. G. A. Paris, Phys. Rev. Lett. 108, 260403 (2012).

[21] C. Gehrke, J. Sperling, and W. Vogel, Phys. Rev. A 86, 052118 (2012).

[22] D. Buono, G. Nocerino, V. D’Auria, A. Porzio, S. Olivares, and M. G. A. Paris, J. Opt. Soc. Am. B 27, A110 (2010); D. Buono, G. Nocerino, A. Porzio, and S. Solimeno, Phys. Rev. A 86, 042308 (2012).

[23] R. L. Hudson, Rep. Math. Phys. 6, 249 (1974).

[24] T. Brocker and R. F. Werner, J. Math. Phys. 36, 62 (1995).

[25] A. Mandilara, E. Karpov, and N. J. Cerf, Phys. Rev. A 79, 062302 (2009).

[26] A. Mari and J. Eisert, Phys. Rev. Lett. 109, 230503 (2012). 
[27] V. Veitch, N. Wiebe, C. Ferrie, and J. Emerson, New J. Phys. 15, 013037 (2013).

[28] M. G. Genoni, M. G. A. Paris, and K. Banaszek, Phys. Rev. A 76, 042327 (2007).

[29] M. G. Genoni, M. G. A. Paris, and K. Banaszek, Phys. Rev. A 78, 060303 (2008).

[30] M. G. Genoni and M. G. A. Paris, Phys. Rev. A 82, 052341 (2010).

[31] M. Barbieri, N. Spagnolo, M. G. Genoni, F. Ferreyrol, R. Blandino, M. G. A. Paris, P. Grangier, and R. Tualle-Brouri, Phys. Rev. A 82, 063833 (2010).

[32] A. Allevi, A. Andreoni, F. A. Beduini, M. Bondani, M. G. Genoni, S. Olivares, and M. G. A. Paris, Europhys. Lett. 92, 20007 (2010).

[33] A. Allevi, A. Andreoni, M. Bondani, M. G. Genoni, and S. Olivares, Phys. Rev. A 82, 013816 (2010).

[34] S. M. Barnett and P. M. Radmore, Methods in Theoretical Quantum Optics (Clarendon Press, Oxford, UK, 1997).

[35] R. Filip and L. Mista, Phys. Rev. Lett. 106, 200401 (2011).
[36] M. G. Genoni, M. L. Palma, T. Tufarelli, S. Olivares, M. S. Kim, and M. G. A. Paris, Phys. Rev. A 87, 062104 (2013).

[37] M. Ježek, I. Straka, M. Mičuda, M. Dušek, J. Fiurašek, and R. Filip, Phys. Rev. Lett. 107, 213602 (2011).

[38] M. Ježek, A. Tipsmark, R. Dong, J. Fiurašek, L. Mišta, Jr., R. Filip, and U. L. Andersen, Phys. Rev. A 86, 043813 (2012).

[39] A. Predojevic, M. Jezek, T. Huber, H. Jayakumar, T. Kauten, G. S. Solomon, R. Filip, and G. Weihs, Opt. Express 22, 4789 (2014).

[40] M. S. Kim, J. Phys. B: At. Mol. Opt. Phys. 41, 133001 (2008).

[41] K. E. Cahill and R. J. Glauber, Phys Rev. 177, 1882 (1969); 177, 1857 (1969).

[42] K. Husimi, Proc. Phys. Soc. Japan 22, 264 (1940).

[43] M. L. Palma, J. Stammers, M. G. Genoni, T. Tufarelli, S. Olivares, M. S. Kim, and M. G. A. Paris, Phys. Scr. T 160, 014035 (2014).

[44] M. G. A. Paris, Phys. Rev. A 53, 2658 (1996).

[45] When $n \gtrsim 3 \times 10^{15}$, our numerics become unstable and no conclusive results have been obtained. 